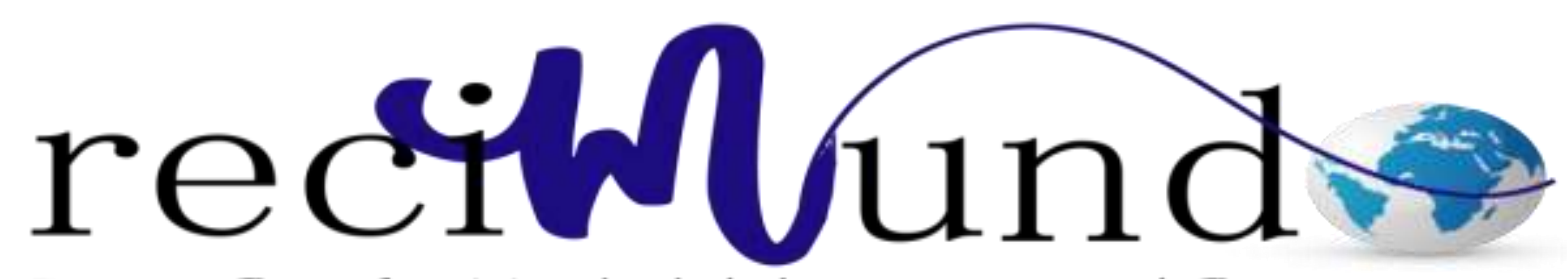

Revista Cientifica Mundo de la Investigación y el Conocimiento

María Belén Palacios Mera ${ }^{\text {a; }}$ Cristhian Wladimir Rivera Baque ${ }^{\text {b; }}$ José Leonardo Castro Zambrano ${ }^{\mathrm{c}}$; Biuthy Lisseth Carvajal Zambrano ${ }^{\mathrm{d}}$

Diagnóstico Clínico e Imagenológico de Patologías Abdominales: Páncreas, Hígado y Vías Biliares

Clinical and Imaging Diagnosis of Abdominal Pathologies: Pancreas, Liver and Bile Ducts

Revista Científica Mundo de la Investigación y el Conocimiento. Vol. 3 núm. 4., diciembre, ISSN: 2588-073X, 2019, pp. 399-415

DOI: $10.26820 /$ recimundo/3.(4).diciembre.2019.399-415

URL: http://recimundo.com/index.php/es/article/view/676

Código UNESCO: 3205 Medicina Interna

Tipo de Investigación: Artículo de Revisión

(C) RECIMUNDO; Editorial Saberes del Conocimiento, 2019

Recibido: 15/09/2019

Aceptado: 23/11/2019

Publicado: 30/12/2019

Correspondencia: karinaborjaa@gmail.com

a. Médico Cirujana; Investigadora Independiente; Guayaquil, Ecuador; bebe-01@ hotmail.com

b. Médico Cirujano; Investigador Independiente; Guayaquil, Ecuador; cristhianwladimir_0127@ hotmail.com

c. Médico Cirujano; Investigador Independiente; Guayaquil, Ecuador; leonardo10_gato@hotmail.com

d. Médico Cirujana; Investigadora Independiente; Guayaquil, Ecuador; biuthycarvajal@ gmail.com 


\section{Diagnóstico Clínico e Imagenológico de Patologías Abdominales: Páncreas, Hígado y Vías Biliares}

Vol. 3, núm. 4., (2019)

María Belén Palacios Mera; Cristhian Wladimir Rivera Baque; José Leonardo Castro Zambrano; Biuthy Lisseth Carvajal Zambrano

\section{RESUMEN}

El abdomen agudo representa un cuadro grave en todo el mundo, el cual constituye una emergencia médica. Se encuentra caracterizado por una serie de síntomas y signos, cuya localización está en la región abdominal. En el ámbito médico, es usual que estos tipos de pacientes sean motivo de sospecha que la causa que la origina la emergencia sea una patología abdominal, las más importantes están relacionadas con el páncreas, el hígado y las vías biliares. Dichas patologías pueden ser progresivas y perjudiciales para estos órganos e incluso para la vida del paciente. El presente estudio tiene como objetivo plasmar lo relativo al diagnóstico clínico e imagenológico de las patologías abdominales más importantes relacionadas con el hígado, el páncreas y las vías biliares. El diseño de investigación que se llevó a cabo es de tipo documental o bibliográfico. Las herramientas de diagnóstico están constituidas por el diagnóstico clínico (anamnesis y exploración física) y las pruebas complementarias: laboratorio e imagenología. Para el presente trabajo no puntualizaremos las pruebas de laboratorio. Entre las principales pruebas complementarias de imágenes se encuentran: rayos $\mathrm{X}$, tomografías computarizadas, estudios de medicina nuclear, imágenes por resonancia magnética y ecografías. El diagnóstico temprano y certero de las patologías abdominales: páncreas, hígado y vías biliares, resulta determinante para prescribir un tratamiento efectivo que minimice y/o evite complicaciones y mejore el estado de salud general del paciente, para este diagnóstico el conocimiento que tenga el médico de la enfermedad y las herramientas exploratorias son fundamentales para precisar el cuadro clínico presente.

Palabras Claves: Diagnóstico; Clínico; Imagenológico; Patología; Abdominal 


\title{
Diagnóstico Clínico e Imagenológico de Patologías Abdominales: Páncreas, Hígado y Vías Biliares
}

Vol. 3, núm. 4., (2019) María Belén Palacios Mera; Cristhian Wladimir Rivera Baque; José Leonardo Castro Zambrano;

Biuthy Lisseth Carvajal Zambrano

\begin{abstract}
The acute abdomen represents a serious condition worldwide, which constitutes a medical emergency. It is characterized by a series of symptoms and signs whose location is in the abdominal region. In the medical field, it is usual that these types of patients are a cause for suspicion that the cause of the emergency is an abdominal pathology, the most important are related to the pancreas, liver and bile ducts. Such pathologies can be progressive and harmful to these organs and even to the patient's life. The present study aims to capture the relative clinical and imaging diagnosis of the most important abdominal pathologies related to the liver, pancreas and bile ducts. The research design that was carried out is documentary or bibliographic. The diagnostic tools are made up of the clinical diagnosis (anamnesis and physical examination) and the complementary tests: laboratory and imaging. For this work we will not specify laboratory tests. Among the main complementary tests of images are: X-rays, CT scans, nuclear medicine studies, magnetic resonance imaging and ultrasound. The early and accurate diagnosis of abdominal pathologies: pancreas, liver and bile ducts, is decisive to prescribe an effective treatment that minimizes and / or avoids complications and improves the patient's general health, for this diagnosis the doctor's knowledge of the disease and exploratory tools are essential to specify the present clinical picture.
\end{abstract}

Keywords: Diagnostic; Clinical; Imaging; Pathology; Abdominal 


\section{Diagnóstico Clínico e Imagenológico de Patologías Abdominales: Páncreas, Hígado y Vías Biliares}

Vol. 3, núm. 4., (2019)

María Belén Palacios Mera; Cristhian Wladimir Rivera Baque; José Leonardo Castro Zambrano; Biuthy Lisseth Carvajal Zambrano

\section{Introducción.}

El abdomen agudo representa un cuadro grave que constituye una emergencia médica, la cual se se caracteriza por una serie de síntomas y signos, cuya localización está en la región abdominal. En el ámbito médico, es usual que estos tipos de pacientes sean motivo de sospecha que la causa que la origina la emergencia sea una patología abdominal, la cual puede ser progresiva y perjudicial para alguno de los órganos de la cavidad abdominal, incluso para la vida del paciente.

Para Kamin et al., 2003; Cartwright \& Knudson 2008 citados por Buitrago-Jaramillo (2019) el abdomen agudo, también conocido como dolor abdominal de inicio súbito, se presenta en los servicios de urgencia como una causa de consulta desafiante y muy frecuente. Asimismo, destacan que a pesar de que "la mayoría de las veces el dolor abdominal se origina en un problema benigno y autolimitado, hasta un $10 \%$ de los pacientes, tendrán una etiología severa, que amenazará su vida y requerirá cirugía”. (p. 1).

El interrogatorio realizado por un médico con experiencia es un proceso activo y de fundamental importancia para el diagnóstico temprano y certero de la patología causante. En él deben considerarse una gama de posibilidades diagnósticas, con la finalidad de eliminar de manera sistemática aquellas patologías menos probables. Es importante observar las consideraciones detalladas de la ubicación del dolor abdominal, el modo de inicio y la progresión, asimismo, su manifestación más específica, ya que estos aspectos constituyen la base para elaborar la lista preliminar que permitirá el diagnóstico diferencial. (Doherty, 2011). 


\section{Diagnóstico Clínico e Imagenológico de Patologías Abdominales: Páncreas, Hígado y Vías Biliares}

Vol. 3, núm. 4., (2019) María Belén Palacios Mera; Cristhian Wladimir Rivera Baque; José Leonardo Castro Zambrano;

Biuthy Lisseth Carvajal Zambrano

Veléz-Hurtado \& Peinado-Acevedo (2018) refieren que las causas del dolor abdominal agudo pueden ser múltiples, generalmente son consecuencia de patologías abdominales tales como hepatitis virales y las de origen no infeccioso como la hepatitis alcohólica o la autoinmune, neoplasias malignas de hígado y vías biliares, alteraciones del bazo, la esplenomegalia, el infarto esplénico, entre otros. No obstante, pueden tener un origen extra abdominal, por ejemplo, es frecuente que los pacientes con insuficiencia cardíaca presenten dolor abdominal, las neumonías basales o derrame pleural, así como la irritación diafragmática puede ocasionar dolor abdominal. (p. 62).

El presente estudio tiene como objetivo plasmar lo relativo al diagnóstico clínico e imagenológico de las patologías abdominales más importantes relacionadas con el hígado, el páncreas y las vías biliares, dada su importancia para que el profesional de la salud identifique la enfermedad a tiempo, valiéndose de las principales herramientas (la anamnesis, el examen físico y los complementarios: imagenología) que le permitirán definir el cuadro clínico presente.

\section{Materiales y Métodos.}

Para el desarrollo de la presente investigación se utilizaron computadores personales con conexión a internet, con la finalidad de recolectar el material bibliográfico digital relacionado con el diagnóstico clínico e imagenológico de las patologías abdominales más importantes del páncreas, hígado y las vías biliares. En virtud de lo anterior, este trabajo encaja dentro de la clasificación de diseño de investigación documental o bibliográfico. Se limitó la investigación a la búsqueda y revisión sistemática de material bibliográfico accesible mediante el uso de diversas 


\section{Diagnóstico Clínico e Imagenológico de Patologías Abdominales: Páncreas, Hígado y Vías Biliares}

Vol. 3, núm. 4., (2019)

María Belén Palacios Mera; Cristhian Wladimir Rivera Baque; José Leonardo Castro Zambrano; Biuthy Lisseth Carvajal Zambrano

bases de datos, tales como: MedlinePlus, Mayo Clinic, PubMed, Biblioteca Virtual de la Salud (BVS), SciELO y Sociedad Americana contra el Cáncer.

Los descriptores usados para realizar la búsqueda fueron: “diagnóstico clínico e imagenológico de patologías abdominales", "patologías abdominales"; "dolor agudo abdominal”, “diagnóstico de patologías pancreáticas”, “diagnóstico de patologías del hígado” y "diagnóstico de patologías de las vías biliares”, posteriormente los resultados de la búsqueda fueron filtrados bajo los siguientes criterios: idioma español, relevancia, correlación temática y fecha de publicación en los últimos ocho años.

\section{Resultados.}

\section{Diagnóstico Clínico (Anamnesis y exploración física)}

Una manera práctica de definir la anamnesis es como la información que un paciente le suministra al médico tratante con la finalidad de elaborar su historia clínica. Esta permite que el paciente exponga hechos que el médico sería incapaz de percibir con la simple exploración física. Es un proceso en el que la comunicación correcta entre el médico y el paciente es crucial para la precisión de los datos recolectados y una buena base para la presunción de determinada patología y el diagnóstico de la misma, en este interrogatorio también pueden intervenir los familiares, cuidadores o personas cercanas al paciente. También está compuesta por los signos que el profesional de la salud pueda detectar, así como de los síntomas que el paciente manifieste.

Cada especialista realiza la anamnesis de manera distinta de acuerdo a cada necesidad, una anamnesis llevada a cabo por un internista contendrá un esquema de preguntas diferente a la de un 


\section{Diagnóstico Clínico e Imagenológico de Patologías Abdominales: Páncreas, Hígado y Vías Biliares}

Vol. 3, núm. 4., (2019) María Belén Palacios Mera; Cristhian Wladimir Rivera Baque; José Leonardo Castro Zambrano; Biuthy Lisseth Carvajal Zambrano

cardiólogo, cada uno estará orientado al área de su especialidad y sobre el motivo de la consulta que exprese el paciente.

No obstante, existen normas generales a seguir en todos los casos que incluyen la preparación del espacio de la entrevista, el saludo personalizado al paciente, en el interrogatorio la primera pregunta será la causa de la consulta, los síntomas, la duración de estos, antecedentes de enfermedades, alergias, procedimientos quirúrgicos practicados, entre otros, que permitan en una primera fase orientar el examen físico, y sobre los hallazgos de este (signos visibles o palpables) realizar nuevas preguntas que permitan precisar el diagnóstico y tratamiento del paciente.

Por su parte, el examen físico o exploración física, consiste en el estudio del cuerpo del paciente para determinar determinada patología. Un examen físico se comprende en líneas generales de:

- Inspección (observar el cuerpo).

- Palpación (sentir el cuerpo con los dedos o las manos).

- Auscultación (escuchar los sonidos).

- Percusión (producir sonidos, generalmente dando golpes suaves en áreas específicas del cuerpo). (Enciclopedia Médica ADAM, 2019).

De lo anterior se deriva la relación estrecha que existe entre la anamnesis y la exploración física, siendo para algunos autores, en su conjunto, la piedra angular del diagnóstico clínico de una enfermedad. 


\section{Diagnóstico Clínico e Imagenológico de Patologías Abdominales: Páncreas, Hígado y Vías Biliares}

Vol. 3, núm. 4., (2019)

María Belén Palacios Mera; Cristhian Wladimir Rivera Baque; José Leonardo Castro Zambrano; Biuthy Lisseth Carvajal Zambrano

\section{Patologías Pancreáticas}

Para el diagnóstico clínico de las patologías pancreáticas el estudio debe de incluir una adecuada anamnesis, que indague acerca del consumo de alcohol y tabaco del paciente, así como de enfermedades asociadas, que incluya antecedentes de enfermedades pancreáticas y autoinmunes.

Una de las patologías más frecuentes e importantes del páncreas es la pancreatitis aguda, se trata de la inflamación aguda del páncreas, en algunos casos incluye los tejidos adyacentes. Su gravedad puede clasificarse en leve, moderada o grave. El diagnóstico está fundamentado en la presentación clínica y los niveles séricos de amilasa y lipasa. Es importante que el médico sospeche de esta patología en todos los casos de dolor abdominal intenso, más aún, en aquellos pacientes cuyo consumo de alcohol es en significativas cantidades, o en aquellos casos en que estos pacientes presentan un diagnóstico previo de litiasis biliar. En líneas generales, el diagnóstico de la pancreatitis aguda se establece cuando al menos 2 de las siguientes manifestaciones se presentan:

- Dolor abdominal compatible con enfermedad

- Amilasa y/o lipasa sérica > 3 veces el límite superior normal

- Hallazgos característicos en las imágenes abdominales. (Bansal, 2017).

Otra enfermedad importante en todo el mundo, a nivel de este órgano es el Cáncer de Páncreas. Una vez diagnosticado, se requieren más pruebas a los fines de determinar la extensión, etapa o estadio en que se encuentra el cáncer. Es importante para el médico incluir en el interrogatorio preguntas relativas a sus antecedentes médicos, síntomas, posibles factores de 


\section{Diagnóstico Clínico e Imagenológico de Patologías Abdominales: Páncreas, Hígado y Vías Biliares}

Vol. 3, núm. 4., (2019) María Belén Palacios Mera; Cristhian Wladimir Rivera Baque; José Leonardo Castro Zambrano; Biuthy Lisseth Carvajal Zambrano

riesgo, incluyendo tabaquismo y antecedentes familiares. Los cánceres de páncreas en ocasiones causan crecimiento del hígado o de la vesícula biliar que pueden ser palpados durante el examen físico. En el examen físico se revisará la piel y la parte blanca de los ojos para verificar ictericia (color amarillento) en el paciente. (Sociedad Americana contra el Cáncer, 2019).

\section{Patologías del Hígado}

El hígado es el órgano de mayor tamaño dentro del cuerpo, entre sus funciones se encuentran cooperar en la digestión de los alimentos, almacenamiento de energía y eliminación de toxinas. Por tal razón, las patologías del hígado son una de las más importantes patologías abdominales.

Los síntomas de la enfermedad hepática pueden ser variados, incluyen hinchazón del abdomen y las piernas, presencia de moretones, cambios en el color de las heces y la orina, y piel y ojos amarillentos o ictericia. En ocasionas es asintomática. Las pruebas complementarias de imagen y de función hepática son importantes no solo para el diagnóstico de la enfermedad sino para comprobar si hay daño en el hígado. (Institutos Nacionales de la Salud de los Estados Unidos - NIH, 2019).

Una patología importante del hígado es la cirrosis, se trata de una fibrosis hepática tardía que ocasiona una distorsión generalizada de la estructura hepática normal. El diagnóstico comprende:

- Hepatograma, pruebas de coagulación, hemograma completo y pruebas serológicas para virus. 


\section{Diagnóstico Clínico e Imagenológico de Patologías Abdominales: Páncreas, Hígado y Vías Biliares}

Vol. 3, núm. 4., (2019)

María Belén Palacios Mera; Cristhian Wladimir Rivera Baque; José Leonardo Castro Zambrano; Biuthy Lisseth Carvajal Zambrano

- A veces, biopsia (p. ej., cuando las pruebas clínicas y no invasivas no son concluyentes o cuando los resultados de la biopsia pueden cambiar el tratamiento)

- A veces, elastografía por ecografía o por resonancia magnética.

- Identificación de la causa sobre la base de la evaluación clínica, pruebas de rutina para causas frecuentes y pruebas selectivas para causas menos frecuentes. (Civan, 2018).

Otra patología importante del hígado es el hígado graso, no es fácil de diagnosticar dado que con frecuencia no presenta síntomas. Para su diagnóstico, el médico debe realizar una buena anamnesis y exploración física y apoyarse en pruebas complementarias, tales como análisis de sangre y de imágenes, incluso, en ocasiones puede ameritar una biopsia. Es importante en el interrogatorio incluir preguntas acerca del consumo de alcohol para diferenciar si la grasa en el hígado es un signo de enfermedad de hígado graso por alcohol o hígado graso no alcohólico. Asimismo, es importante indagar acerca de las medicinas que toma el paciente, a los fines de puntualizar si el hígado graso es consecuencia de un medicamento. Durante la exploración física, su médico verificará el peso y la talla del paciente, este debe buscar signos de hígado graso, tales como: hígado agrandado (hepatomegalia), signos de cirrosis como ictericia, entre los más destacados. (NIH, 2019).

\section{Patologías de las Vías Biliares}

La litiasis en la vesícula biliar es una de las patologías de salud más importantes que desde remotos tiempos afectan a las personas a nivel mundial. Esta enfermedad es de frecuencia elevada y puede presentar serias complicaciones. Para Almora, Arteaga, Plaza, Prieto, \& Hernández, 


\section{Diagnóstico Clínico e Imagenológico de Patologías Abdominales: Páncreas, Hígado y Vías Biliares}

Vol. 3, núm. 4., (2019) María Belén Palacios Mera; Cristhian Wladimir Rivera Baque; José Leonardo Castro Zambrano;

Biuthy Lisseth Carvajal Zambrano

(2012) en vista de que la mayoría de los casos de vesícula biliar o de vías biliares el paciente es asintomático, la exploración física, por lo general, es normal. Por medio de la anamnesis se puede llegar a la sospecha clínica y es a través de pruebas complementarias es que se precisa el diagnóstico. Asimismo, los autores resaltan la importancia de realizar el diagnóstico diferencial con otras enfermedades tales como: "úlcera péptica, dolor torácico, reflujo gastroesofágico, dispepsia no ulcerosa, colon irritable y hepatitis. Estos procesos son frecuentes en la población general y pueden darse en un paciente con litiasis biliar, sin que la clínica esté relacionada con los cálculos”. (p. 210).

Imagenología

Este diagnóstico se realiza observando el interior del cuerpo del paciente con la finalidad de buscar indicios sobre una afección médica. En la actualidad una gran variedad de aparatos y de técnicas pueden crear imágenes de las diferentes estructuras, incluso por medio de estas se puede observar actividades dentro del cuerpo que permiten una comprensión más precisa del cuadro clínico presente. El tipo de imagen seleccionado por el médico dependerá de los síntomas y de la zona del cuerpo que deba ser examinada (datos recolectados en la anamnesis y exploración física). Algunos de estos estudios pueden ser:

\section{- Rayos X}

- Tomografías computarizadas

- Estudios de medicina nuclear

- Imágenes por resonancia magnética 


\section{Diagnóstico Clínico e Imagenológico de Patologías Abdominales: Páncreas, Hígado y Vías Biliares}

Vol. 3, núm. 4., (2019)

María Belén Palacios Mera; Cristhian Wladimir Rivera Baque; José Leonardo Castro Zambrano; Biuthy Lisseth Carvajal Zambrano

- Ecografías. (NIH, 2019).

\section{Patologías Pancreáticas}

La tomografía computarizada (TC) con contraste IV se utiliza en los casos en que el diagnóstico de pancreatitis aguda sea incierto o para descartar otras causas de los síntomas. Asimismo, una vez que se diagnostica pancreatitis, es frecuente el uso de una TC con la finalidad de identificar complicaciones de la enfermedad tales como necrosis, colección líquida o pseudoquistes. "El tejido pancreático necrosado no mejora después de que se administra contraste IV y puede no aparecer en la TC hasta 48 a 72 h después del inicio de la pancreatitis aguda". (Bansal, 2017).

Otro tipo de tomografía es la tomografía computarizada de energía dual (TCED). Al respecto, Baleato, García, Luna, Domínguez, \& Vilanova (2016) la definen como:

La adquisición de imágenes con una combinación de distintos kilos voltajes (la más utilizada es $80 \mathrm{kV}$ y $140 \mathrm{kV}$ ). Dado que la absorción de rayos X depende de la energía del haz y que la atenuación de los distintos elementos químicos es diferente a $80 \mathrm{kV}$ a $140 \mathrm{kV}$, esto permite clasificarlos basándose en el análisis de la diferencia de atenuación con cada espectro de energía. La TCED permitiría mejorar la detección del adenocarcinoma de páncreas. (p. 452).

Para el diagnóstico de la Pancreatitis Las pruebas y los procedimientos que se usan para diagnosticar la incluyen lo siguiente: 


\section{Diagnóstico Clínico e Imagenológico de Patologías Abdominales: Páncreas,}

Hígado y Vías Biliares

Vol. 3, núm. 4., (2019) María Belén Palacios Mera; Cristhian Wladimir Rivera Baque; José Leonardo Castro Zambrano; Biuthy Lisseth Carvajal Zambrano

- Tomografía computarizada (TC) para detectar cálculos biliares y evaluar la inflamación del páncreas.

- Ecografía abdominal para detectar cálculos biliares e inflamación del páncreas

- Ecografía endoscópica para detectar inflamación y bloqueos en el conducto pancreático o en las vías biliares

- Resonancia magnética (RM) para detectar anomalías en la vesícula, el páncreas y los conductos. (Mayo Clinic, 2019).

Las imágenes por resonancia magnética (MRI) también son usadas en el diagnóstico de patologías pancreáticas, estas utilizan ondas de radio e imanes potentes en lugar de rayos X con la finalidad de crear imágenes detalladas de las partes del cuerpo. Es importante destacar que, pese a que la mayoría de los médicos prefieren examinar el páncreas por medio de una TC, la MRI también es una opción, especialmente en el caso de algunos tipos especiales de este estudio en personas que podrían tener cáncer de páncreas o presenten mayor riesgo de padecerlo. Estos estudios especiales incluyen la colangiopancreatografía MR (MRCP) la cual puede ser usada en la observación de los conductos biliares y pancreáticos. Asimismo, puede ser usada la angiografía por MR (MRA) con la finalidad de observar los vasos sanguíneos. (Sociedad Americana contra el Cáncer, 2019).

\section{Patologías del Hígado}

El elastografía por ecografía y la elastografía por resonancia magnética son utilizados para la detección precoz de la cirrosis, en aquellos casos en los que los hallazgos de otros estudios de 


\section{Diagnóstico Clínico e Imagenológico de Patologías Abdominales: Páncreas, Hígado y Vías Biliares}

Vol. 3, núm. 4., (2019)

María Belén Palacios Mera; Cristhian Wladimir Rivera Baque; José Leonardo Castro Zambrano; Biuthy Lisseth Carvajal Zambrano

por imágenes convencionales no son concluyentes y la hipertensión portal no es evidente. Una de las características de la cirrosis avanzada en la ecografía es un hígado pequeño nodular. La ecografía puede identificar además la hipertensión portal y la ascitis. Otro estudio imagenológico utilizado en la detección o diagnóstico de las patologías hepáticas son las tomografías computarizadas (TC), las cuales pueden detectar la morfología nodular, no obstante, no ofrece las ventajas que aporta la ecografía. "La gammagrafía hepática con radionúclidos como tecnecio-99m de azufre coloidal podría revelar una captación hepática irregular y un aumento de la captación en el bazo y la médula ósea". (Civan, 2018).

\section{Patologías de las Vías Biliares}

Para diagnosticar las patologías de la vesícula y de las vías biliares pueden ser usados estudios de imágenes como la ecografía transabdominal, la cual es muy precisa para diagnosticar cálculos biliares > 2 mm de diámetro, (sensibilidad > 95\%) para la detección de cálculos biliares. En casos de cálculos más pequeños, la ecografía por vía endoscópica es una buena opción, ya que puede detectar cálculos tan pequeños como de $0,5 \mathrm{~mm}$ (microlitiasis) en la vesícula biliar o las vías biliares. La ecografía transabdominal y por vía endoscópica es ideal para identificar "barro biliar" (una mezcla de material particulado y bilis). (Orfanidis, 2017).

\section{Conclusiones.}

Un diagnóstico temprano y preciso de las patologías abdominales, en especial las relacionadas con el páncreas, el hígado y las vías biliares, puede evitar serias complicaciones e incluso pueden salvar la vida del paciente. La falla en el diagnóstico puede ser considerada como 


\section{Diagnóstico Clínico e Imagenológico de Patologías Abdominales: Páncreas, Hígado y Vías Biliares}

Vol. 3, núm. 4., (2019) María Belén Palacios Mera; Cristhian Wladimir Rivera Baque; José Leonardo Castro Zambrano;

Biuthy Lisseth Carvajal Zambrano

negligencia común, el fracaso del médico en su diagnóstico genera un tratamiento ineficáz, que puede acarrear que el paciente sufra daños irreversibles e incluso mortales.

En tal sentido, el conocimiento del médico de la enfermedad y las herramientas de diagnóstico son fundamentales para precisar el cuadro clínico presente. Estas herramientas de diagnóstico están constituidas por el diagnóstico clínico (anamnesis y exploración física), laboratorio e imagenología.

La comunicación médico paciente es fundamental, un buen abordaje del paciente en el motivo de su consulta, los síntomas que lo preocupan, su duración, entre otros, contribuye a orientar el resto de los estudios para precisar el cuadro clínico presentado. La anamnesis puede orientar el examen físico, en donde el médico revisa, palpa, observa, ausculta y percute el cuerpo del paciente y esta orientación lo lleva a seguir interrogando y ordenar las pruebas complementarias pertinentes a cada caso.

El diagnóstico imagenológico de las patologías abdominales es determinante para establecer la patología de la que se sospecha y la gravedad del daño que ha ocasionado al órgano abdominal que la padece. En la actualidad los avances tecnológicos ponen a la disposición del área médica una serie de aparatos y de técnicas cada vez más sofisticadas, que permiten crear imágenes más claras para una mayor comprensión del cuadro clínico presente. Entre los principales se encuentran: rayos X, tomografías computarizadas, estudios de medicina nuclear, imágenes por resonancia magnética y ecografías. 


\section{Diagnóstico Clínico e Imagenológico de Patologías Abdominales: Páncreas, Hígado y Vías Biliares}

Vol. 3, núm. 4., (2019)

María Belén Palacios Mera; Cristhian Wladimir Rivera Baque; José Leonardo Castro Zambrano; Biuthy Lisseth Carvajal Zambrano

Independientemente de la tecnología disponible, un buen diagnóstico de las patologías abdominales: de páncreas, hígado y vías biliares, dependerá del conocimiento y la experiencia que tenga el médico acerca de estas patologías y de las herramientas para explorar al paciente y precisar con rapidez un diagnóstico certero para prescribir un tratamiento efectivo que minimice y/o evite complicaciones y mejore el estado de salud general del paciente.

\section{Bibliografía.}

Almora, C., Arteaga, Y., Plaza, T., Prieto, Y., \& Hernández, Z. (2012). Diagnóstico clínico y epidemiológico de la litiasis vesicular. Revisión bibliográfica. Revista de Ciencias Médicas, 16(1), 200-214.

Baleato, S., García, R., Luna, A., Domínguez, M., \& Vilanova, J. (Septiembre de 2016). Imagen funcional de la patología pancreática. (S. E. Médica, Ed.) Radiología, 60(6), 451-464.

Bansal, R. (Febrero de 2017). Pancreatitis Aguda. Obtenido de Manual MSD: https://www.msdmanuals.com/es-ve/professional/trastornosgastrointestinales/pancreatitis/pancreatitis-aguda

Buitrago-Jaramillo, J. (2019). Abdomen Agudo. Conferencia. Recuperado el 01 de Diciembre de 2019, de https://www.researchgate.net/publication/331181833_Abdomen_Agudo

Civan, J. M. (Febrero de 2018). Cirrosis. Obtenido de Manual MSD: https://www.msdmanuals.com/es-ve/professional/trastornos-hep\%C3\%A1ticos-ybiliares/fibrosis-y-cirrosis/cirrosis

Doherty, G. M. (2011). Abdomen agudo. En G. M. Doherty, Diagnóstico y tratamiento quirúrgicos (13 e ed., pág. 1130). Mac Graw-Hill- LAnge. Recuperado el 02 de Diciembre de 2019, de https://accessmedicina.mhmedical.com/content.aspx?bookid=1478\&sectionid=10287992 5

Enciclopedia Médica ADAM. (02 de Julio de 2019). Examen físico. Obtenido de MedlinePlus: https://medlineplus.gov/spanish/ency/article/002274.htm

Institutos Nacionales de la Salud de los Estados Unidos - NIH. (13 de Junio de 2019). Enfermedades del hígado. Obtenido de MedlinePlus: https://medlineplus.gov/spanish/liverdiseases.html 


\section{Diagnóstico Clínico e Imagenológico de Patologías Abdominales: Páncreas,}

Hígado y Vías Biliares

Vol. 3, núm. 4., (2019)

María Belén Palacios Mera; Cristhian Wladimir Rivera Baque; José Leonardo Castro Zambrano;

Biuthy Lisseth Carvajal Zambrano

Mayo Clinic. (14 de Diciembre de 2019). Pancreatitis. Obtenido de Mayo Clinic: https://www.mayoclinic.org/es-es/diseases-conditions/pancreatitis/diagnosistreatment/drc-20360233

NIH. (15 de Julio de 2019). Diagnóstico por imágenes. Obtenido de MedlinePlus: https://medlineplus.gov/spanish/diagnosticimaging.html

NIH. (24 de Junio de 2019). Hígado graso. Obtenido de MedlinePlus: https://medlineplus.gov/spanish/fattyliverdisease.html

Orfanidis, N. T. (Febrero de 2017). Pruebas de diagnóstico por la imagen para el hígado y la vesícula biliar. Obtenido de Manual MSD: https://www.msdmanuals.com/es/professional/trastornos-hep\%C3\%A1ticos-ybiliares/pruebas-para-trastornos-hep\%C3\%A1ticos-y-biliares/pruebas-dediagn\%C3\%B3stico-por-la-imagen-para-el-h\%C3\%ADgado-y-la-ves\%C3\%ADculabiliar

Sociedad Americana contra el Cáncer. (11 de Febrero de 2019). Cáncer de Páncreas: Detección $y$ diagnóstico. Obtenido de Sociedad Americana contra el Cáncer: https://www.cancer.org/es/cancer/cancer-de-pancreas/deteccion-diagnosticoclasificacion-por-etapas/como-se-diagnostica.html

Veléz-Hurtado, D., \& Peinado-Acevedo, J. S. (2018). Dolor abdominal en urgencias: la perspectiva del médico internista. XVIII Curso de Actualización en Medicina Interna: Retos Clínicos en Medicina Interna, Universidad de Antioquia, Escuela de Medicina, Antioquia. Recuperado el 03 de Diciembre de 2019, de https://www.researchgate.net/publication/324272557_Dolor_abdominal_la_perspectiva_ del_internista

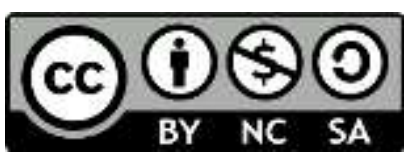

RECONOCIMIENTO-NOCOMERCIAL-COMPARTIRIGUAL

CC BY-NC-SA

ESTA LICENCIA PERMITE A OTROS ENTREMEZCLAR, AJUSTAR Y CONSTRUIR A PARTIR DE SU OBRA CON FINES NO COMERCIALES, SIEMPRE Y CUANDO LE RECONOZCAN LA AUTORÍA Y SUS NUEVAS CREACIONES ESTÉN BAJO UNA LICENCIA CON LOS MISMOS TÉRMINOS. 